\title{
Electronic properties of dislocations in GaN investigated by scanning tunneling microscopy
}

Ph. Ebert, L. Ivanova, S. Borisova, H. Eisele, A. Laubsch, and M. Dähne

Citation: Appl. Phys. Lett. 94, 062104 (2009);

View online: https://doi.org/10.1063/1.3073741

View Table of Contents: http://aip.scitation.org/toc/apl/94/6

Published by the American Institute of Physics

\section{Articles you may be interested in}

Doping modulation in GaN imaged by cross-sectional scanning tunneling microscopy

Applied Physics Letters 94, 162110 (2009); 10.1063/1.3123258

Meandering of overgrown v-shaped defects in epitaxial GaN layers

Applied Physics Letters 105, 012105 (2014); 10.1063/1.4887372

Repulsive interactions between dislocations and overgrown v-shaped defects in epitaxial GaN layers Applied Physics Letters 103, 142105 (2013); 10.1063/1.4823474

Hidden surface states at non-polar GaN (1010) facets: Intrinsic pinning of nanowires

Applied Physics Letters 103, 152101 (2013); 10.1063/1.4823723

Probing defect states in polycrystalline $\mathrm{GaN}$ grown on $\mathrm{Si}(111)$ by sub-bandgap laser-excited scanning tunneling spectroscopy

Journal of Applied Physics 121, 015701 (2017); 10.1063/1.4972563

Evidence of deep traps in overgrown v-shaped defects in epitaxial GaN layers

Applied Physics Letters 103, 062101 (2013); 10.1063/1.4816969

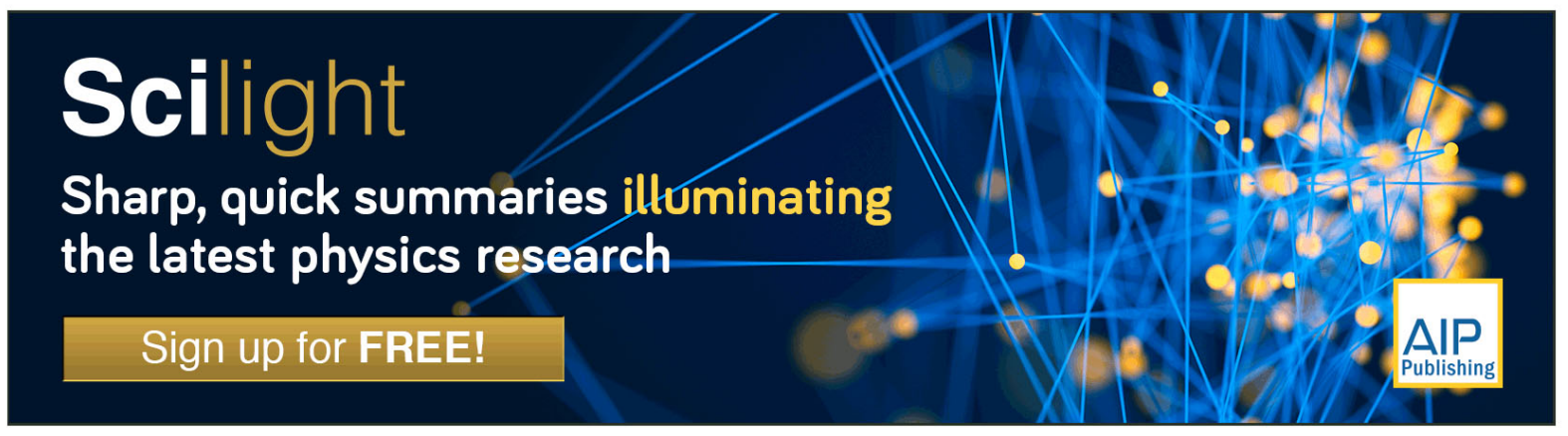




\title{
Electronic properties of dislocations in GaN investigated by scanning tunneling microscopy
}

\author{
Ph. Ebert, ${ }^{1, a)}$ L. Ivanova, ${ }^{2}$ S. Borisova, ${ }^{1}$ H. Eisele, ${ }^{2}$ A. Laubsch, ${ }^{3}$ and M. Dähne ${ }^{2}$ \\ ${ }^{1}$ Institut für Festkörperforschung, Forschungszentrum Jülich GmbH, 52425 Jülich, Germany \\ ${ }^{2}$ Institut für Festkörperphysik, Technische Universität Berlin, Hardenbergstr. 36, 10623 Berlin, Germany \\ ${ }^{3}$ OSRAM Opto-Semiconductors GmbH, Leibnizstrasse 4, 93055 Regensburg, Germany
}

(Received 17 November 2008; accepted 2 January 2009; published online 10 February 2009)

\begin{abstract}
We investigated the type, spatial distribution, line direction, and electronic properties of dislocations in $n$-type $\mathrm{GaN}$ by scanning tunneling microscopy. We found uncharged perfect dislocations with $a / 3\{11 \overline{2} 0\}$ Burgers vectors and negatively charged Shockley partial dislocations with $a / 3\{1 \overline{1} 00\}$ Burgers vectors interconnected by a negatively charged stacking fault. The charges are traced to different charge transfer levels associated with the particular core structure. (C) 2009 American Institute of Physics. [DOI: 10.1063/1.3073741]
\end{abstract}

Group-III nitrides developed rapidly toward the materials of choice for green to ultraviolet optoelectronics. However, $\mathrm{GaN}$ devices can only be produced successfully by epitaxial growth due to the high vapor pressure of nitrogen. Unfortunately, no well suited lattice- and thermal-matched substrates are available for GaN epitaxy, leading to strain and huge dislocation densities. ${ }^{1}$ In order to reduce them, special techniques, such as lateral overgrowth or the insertion of strain reducing layers, are used to concentrate the dislocations in bunches. Despite these efforts, the dislocation density in commercial freestanding GaN wafers is still far above that of zinc blende type III-V semiconductor substrates.

The presence of high dislocation densities is detrimental for optoelectronics because dislocations can act as recombination centers. Therefore, large efforts focused on understanding the electronic properties of the different types of dislocations present in GaN. Unfortunately, thus far the experimental results are inconclusive: dislocations in $n$-type $\mathrm{GaN}$ were found to be either all charged, ${ }^{2,3}$ partially charged depending on the specific type, ${ }^{4-6}$ or uncharged. ${ }^{7}$ In addition, strongly varying charge densities were reported for the same type of edge dislocation. ${ }^{2,8}$ In analogy to the experimental inconsistency, the charges of dislocations are attributed to the reconstruction of the dislocation cores, ${ }^{9-12}$ impurities and point defects, ${ }^{6,7,13,14}$ or the introduction of strain-induced gap states. ${ }^{15}$ One particular problem limiting the insight is that many experiments lack atomic resolution and/or are done on surfaces with surface states in the band gap. Therefore, we investigated dislocations in GaN by charge sensitive atomically resolved scanning tunneling microscopy (STM) using clean cleavage surfaces free of intrinsic surface states in the band gap. ${ }^{16}$ This approach enables us to probe simultaneously the charge state and structure of the dislocations. Thereby we identified two types of dislocations: uncharged perfect and negatively charged dissociated dislocations.

For our experiments we cleaved samples cut from $300 \mu \mathrm{m}$ thick $n$-type $\mathrm{GaN}(0001)$ freestanding wafers in ultrahigh vacuum $\left(<10^{-8} \mathrm{~Pa}\right)$ along $(1 \overline{1} 00)$ planes. The GaN wafers were grown by hydride vapor-phase epitaxy and had a resistivity of about $(0.8-1) \times 10^{-2} \Omega \mathrm{cm}$. The specified

${ }^{a)}$ Electronic mail: p.ebert@fz-juelich.de. threading dislocation density of the samples was (1-2) $\times 10^{7} \mathrm{~cm}^{-2}$. For the scanning tunneling microscopy measurements we used $\mathrm{Pt}_{0.8} \mathrm{Ir}_{0.2}$ tips and acquired the data using a RHK SPM control unit.

Figure 1 shows an overview of the $\mathrm{GaN}(1 \overline{1} 00)$ cleavage surface. The surface consists of atomically flat terraces separated by monoatomic steps. Most of the steps cross the whole image. However, four steps terminate suddenly at dislocations (see arrows). The density of these dislocations was not homogeneous across the whole sample. In most areas no dislocations were found. Only in a localized spot of about $5 \mu \mathrm{m}$ diameter the dislocation density reached values of $1.2 \times 10^{8} \mathrm{~cm}^{-2}$. The spatial localization of dislocations and their very high density indicate that the dislocations form bunches, while the remaining material is free of dislocations.

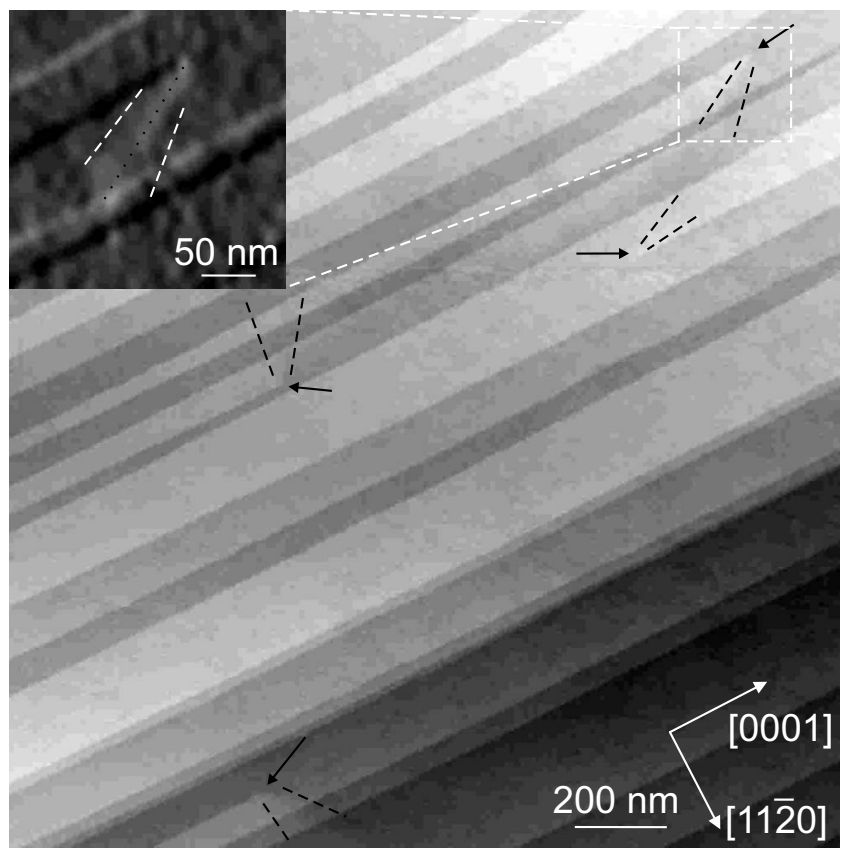

FIG. 1. Empty-state STM image of an area with four dislocations (see arrows) measured at $+4.8 \mathrm{~V}$ and $82 \mathrm{pA}$. The vertical displacement fields of each dislocation are indicated by $\mathrm{v}$-shaped dashed lines. Inset: horizontal gradient illustrating the vertical displacement field of the dislocation in the upper right corner. 


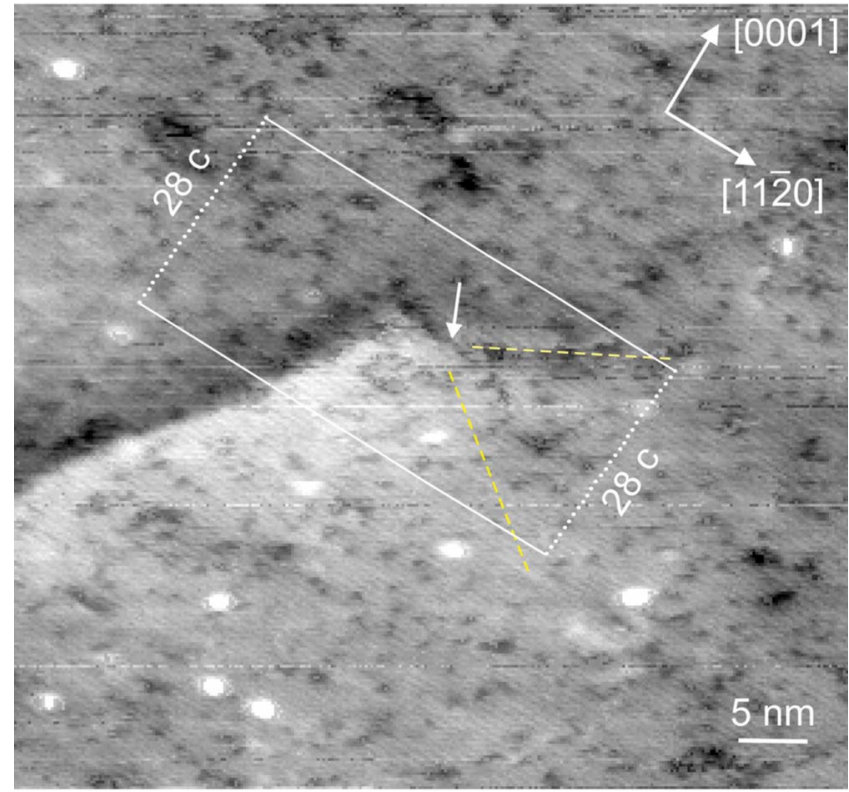

FIG. 2. (Color online) Empty-state STM image of a perfect dislocation with a Burgers vector of type $a / 3\{11 \overline{2} 0\}$ measured at $+4.2 \mathrm{~V}$ and $86 \mathrm{pA}$. The vertical displacement field is indicated by the $\mathrm{v}$-shaped dashed lines. The solid lines and points show that the Burgers vector has no component along the [0001] direction.

During growth of GaN layers on nonlattice matched substrates, dislocations form near the substrate-GaN interface. Their line directions, initially primarily following the [0001] growth direction, ${ }^{17,18}$ may bend with progressing growth toward the side facets as shown, e.g., in Ref. 17. Thereby the dislocation lines may cross the $(1 \overline{1} 00)$ cleavage surface. Since we observed dislocations on our cleavage surface, their lines must have indeed bent away from the [0001] direction. In fact, the component of the dislocation line direction projected on the surface can be extracted from the STM images. Each dislocation induces a displacement field, which is largest close to the dislocation core and decays with increasing distance. If a dislocation line crosses the cleavage surface nonperpendicularly, the displacement field at the surface is largest where the subsurface dislocation core is closest to the surface. The horizontal gradient (inset in Fig. 1) illustrates the resulting v-shaped vertical displacement field of one dislocation. The largest displacements occur along the dotted line. Thus, the dislocation line has a projected component along the same direction. The diagonal contrast lines arise from steps and are not of interest. Analyzing each dislocation in this manner yields that every dislocation has a different line direction (see v-shaped dashed lines in Fig. 1). This indicates that the dislocations form bunches of entangled nonparallel dislocation lines.

We observed two different types of dislocations, perfect nondissociated and dissociated ones. Figure 2 exemplifies a perfect dislocation. The STM image shows the empty dangling bonds above the Ga atoms. ${ }^{16}$ The dislocation core is located at the end of the surface step (arrow). Note that the

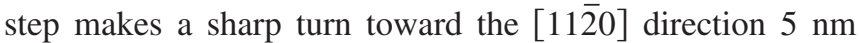
before the dislocation core. The displacement field (v-shaped dashed lines) suggests that the dislocation line is lying in a (0001) plane. We determined the three components of the Burgers vector as follows. First, the dislocation induces a
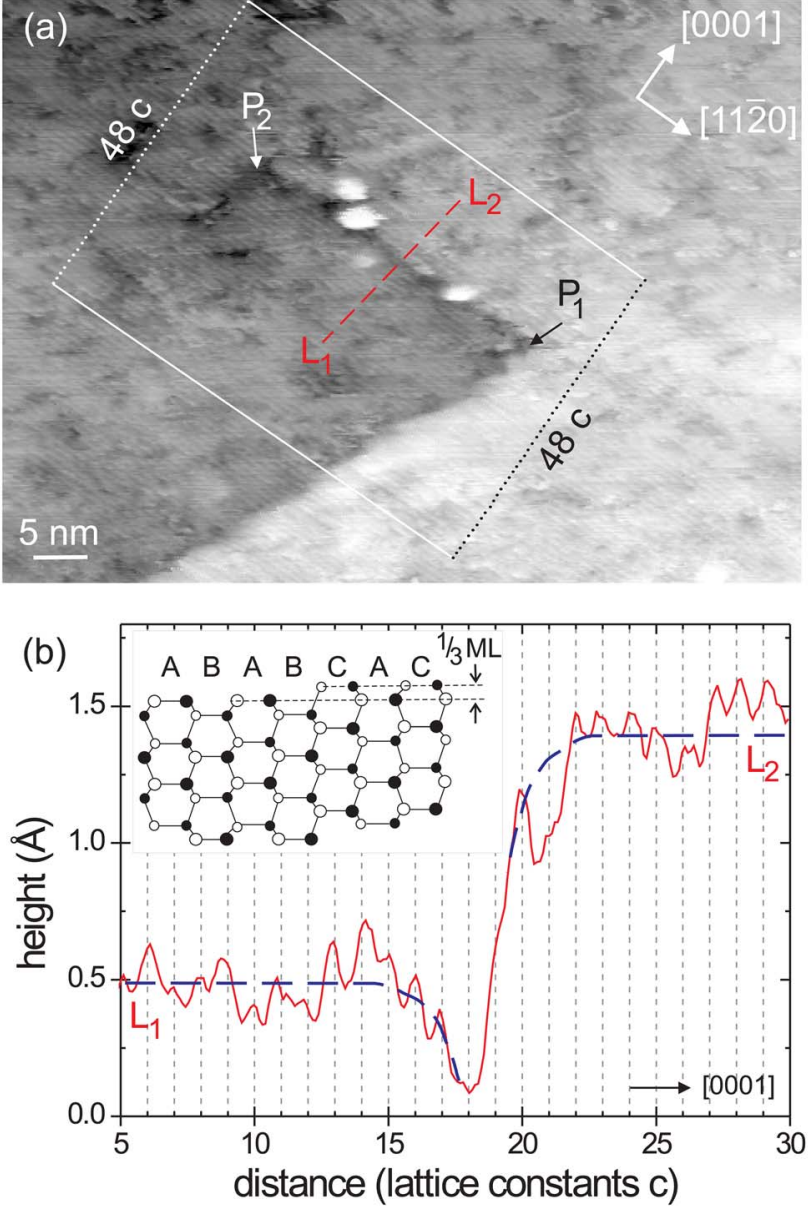

FIG. 3. (Color online) (a) Empty-state STM image of a dislocation with an overall Burgers vector of type $a / 3\{11 \overline{2} 0\}$ dissociated into Shockley partial dislocations $P_{1}$ and $P_{2}$ (measured at $+4.0 \mathrm{~V}$ and $94 \mathrm{pA}$ ). (b) Height profile measured along the dashed line $L_{1}-L_{2}$ in (a). The stacking fault between the two partial dislocations gives rise to a $1 / 3 \mathrm{ML}$ step. The solid lines and points illustrate that the Burgers vectors has no component along the [0001] direction. Inset: schematic of an intrinsic type 2 stacking fault. Black and white circles represent $\mathrm{Ga}$ and $\mathrm{N}$ atoms, respectively.

step with a height of $1 \mathrm{ML}$. Thus, the Burgers vector has a component of $a / 2[1 \overline{1} 00]$ perpendicular to the surface. Second, the separation of the atomic rows in the [11 $\overline{2} 0]$ direction (marked by white lines) equals $28 c$ on both sides of the dislocation core. Thus, the Burgers vector has no component in the [0001] direction. Finally, the step induces not only a component perpendicular to the surface, but also parallel to the atomic rows within the surface plane, because two neighboring $(1 \overline{1} 00)$ planes in the wurtzite structure are offset by $a / 6[11 \overline{2} 0]$. This yields a total Burgers vector of the type $a / 3\{11 \overline{2} 0\}$, tilted $30^{\circ}$ with respect to the surface normal. This is indeed the only possible Burgers vector without any component along the [0001] direction in wurtzite crystals. ${ }^{19}$

Figure 3(a) shows the second type of dislocation, which is dissociated into two partial dislocations marked $P_{1}$ and $P_{2}$. The first partial dislocation core $\left(P_{1}\right)$ is located at the point, where the monoatomic step $(1 \mathrm{ML} \approx 2.75 \AA)$ sharply turns and transforms into a $1 / 3$ ML high step (height $\sim 0.9 \AA$ ). The second partial dislocation is positioned at the end of the $1 / 3$ ML step $\left(P_{2}\right)$. Between both partial dislocations a stacking fault in the (0001) plane is present, which results in the 
1/3 ML height shift visible in the height profile shown in Fig. 3(b). The Burgers vectors are determined as above. The atomic rows in the $[11 \overline{2} 0]$ direction on both sides of the dissociated dislocation marked by white lines are separated everywhere by $48 c$. Thus, the total and partial Burgers vectors have no component in the [0001] direction. The monoatomic step yields again a Burgers vector of the type $a / 3\{11 \overline{2} 0\}$. Dislocations with such Burgers vectors can dissociate into two Shockley partials with Burgers vectors of the type $a / 3\{1 \overline{1} 00\} .{ }^{20}$ With this in mind, the reduction in the step height from 1 to $1 / 3 \mathrm{ML}$ at the partial dislocation $P_{1}$ corresponds to a Burgers vector of $a / 3[1 \overline{1} 00]$ oriented parallel to the surface normal. The partial dislocation $P_{2}$, where the 1/3 ML height shift disappears, has the same type of Burgers vector, but rotated $60^{\circ}$ away from the surface normal.

This identification agrees with the structure of the stacking fault. The height profile in Fig. 3(b) shows not only the height offset of $1 / 3 \mathrm{ML}$, but also the absence of any shift along the [0001] direction of the [1120] oriented atomic rows, because the positions of the maxima on both sides of the stacking fault exactly match the dotted lines. This structure corresponds to a double fault with a stacking sequence ...ABABCACA... (so-called type 2 intrinsic stacking fault), as shown in the inset in Fig. 3(b). Such a stacking fault is connected with Shockley partials, ${ }^{20}$ supporting the above identification of the Burgers vectors and dislocation types.

A close look at the height profile of the stacking fault shows an apparent height depression close to the stacking fault, as indicated by the blue dashed lines in Fig. 3(b). Similar depression zones were observed previously around charged point defects and steps, ${ }^{21}$ where the depression arises from an upward band bending induced by localized negative charges. The band bending reduces the density of empty states in the conduction band available for tunneling at positive voltages. ${ }^{21}$ Therefore, the tip is moved closer to the sample in order to keep the tunnel current constant. This leads to the observed depression zone. Thus, the stacking fault is negatively charged. Similarly, around the partial dislocation cores dark zones are found, suggesting a negative charge of the partial dislocations. In contrast, no dark zones were found at perfect dislocations. In Fig. 2 the surface exhibits a number of charged point defects surrounded by a dark contrast, but at the dislocation core no dark contrast is visible. Thus, perfect dislocations are uncharged on $n$-type GaN. These observations indicate that the charge transfer level $(0 /-)$ is lower in energy for the partial than for the perfect dislocation.

The charges of the partial dislocations are unlikely to be induced by charged impurities/defects attracted in the strain field or strain-induced deep electronic states, because the strain field is reduced for partial dislocations by dissociation as compared to perfect dislocations. Thus, the different charge states are rather originating in the specific core structure of the different dislocations. Indeed, Shockley partial dislocations were predicted to have deep levels in $n$-type $\mathrm{GaN}^{22}$ The charge of the stacking fault itself might arise from additional surface dangling bonds at the edge of the $1 / 3$ ML height shift. However, the stacking fault is a local inser- tion of cubic GaN into the wurtzite structure. Since cubic $\mathrm{GaN}$ has a smaller band gap than wurtzite $\mathrm{GaN}$, the stacking fault will introduce localized gap states. A charging of Shockley partial dislocations and the corresponding stacking fault was also observed in $n$-type GaAs by STM. ${ }^{23}$

In conclusion, we investigated the type, the spatial distribution, the projected line direction, and the electronic properties of dislocations in $n$-type freestanding GaN wafers by STM. The dislocations were found to form localized bunches of entangled nonparallel dislocation lines. Within these bunches perfect dislocations with $a / 3\{11 \overline{2} 0\}$ Burgers vectors were uncharged, while Shockley partials with $a / 3\{1 \overline{1} 00\}$ Burgers vector and the related intrinsic type 2 stacking fault were negatively charged. The observations suggest that the dissociation of dislocations may be responsible for the insertion of detrimental gap states in $n$-type $\mathrm{GaN}$.

The authors thank the Deutsche Forschungsgemeinschaft for financial support.

${ }^{1}$ C. Kisielowski, J. Krüger, S. Ruminov, T. Suski, J. W. Ager III, E. Jones, Z. Liliental-Weber, M. Rubin, E. R. Weber, M. D. Bremser, and R. F Davis, Phys. Rev. B 54, 17745 (1996); P. Gibart, Rep. Prog. Phys. 67, 667 (2004).

${ }^{2}$ J. Cai and F. A. Ponce, Phys. Status Solidi A 192, 407 (2002).

${ }^{3}$ G. Koley and M. G. Spencer, Appl. Phys. Lett. 78, 2873 (2001).

${ }^{4}$ P. J. Hansen, Y. E. Strausser, A. N. Erickson, E. J. Tarsa, P. Kozodoy, E. G. Brazel, J. P. Ibbetson, U. Mishra, V. Narayanamurti, S. P. DenBaars, and J. S. Speck, Appl. Phys. Lett. 72, 2247 (1998).

${ }^{5}$ J. W. P. Hsu, H. M. Ng, A. M. Sergent, and S. N. G. Chu, Appl. Phys. Lett. 81, 3579 (2002).

${ }^{6}$ M. Albrecht, L. J. Weyher, B. Lucznik, I. Grzegory, and S. Porowski, Appl. Phys. Lett. 92, 231909 (2008).

${ }^{7}$ A. Krtschil, A. Dadgar, and A. Krost, Appl. Phys. Lett. 82, 2263 (2003)

${ }^{8}$ D. Cherns and C. G. Jiao, Phys. Rev. Lett. 87, 205504 (2001).

${ }^{9}$ J. Elsner, R. Jones, P. K. Sitch, V. D. Perzag, M. Elstner, Th. Frauenheim, M. I. Heggie, S. Öberg, and P. R. Briddon, Phys. Rev. Lett. 79, 3672 (1997).

${ }^{10}$ Y. Xin, S. J. Pennycook, N. D. Browning, P. D. Nellist, S. Sivananthan, F. Omnès, B. Beaumont, J. P. Faurie, and P. Gibart, Appl. Phys. Lett. 72, 2680 (1998).

${ }^{11}$ A. F. Wright and U. Grossner, Appl. Phys. Lett. 73, 2751 (1998).

${ }^{12}$ J. E. Northrup, Phys. Rev. B 66, 045204 (2002).

${ }^{13}$ A. T. Blumenau, J. Elsner, R. Jones, M. I. Heggie, S. Öberg, T. Frauenheim, and P. R. Briddon, J. Phys.: Condens. Matter 12, 19223 (2000).

${ }^{14}$ I. Arslan, A. Bleloch, E. A. Stach, and N. D. Browning, Phys. Rev. Lett. 94, 025504 (2005).

${ }^{15}$ L. Lymperakis, J. Neugebauer, M. Albrecht, T. Remmele, and H. P. Strunk, Phys. Rev. Lett. 93, 196401 (2004).

${ }^{16}$ L. Ivanova, S. Borisova, H. Eisele, M. Dähne, A. Laubsch, and Ph. Ebert, Appl. Phys. Lett. 93, 192110 (2008); B. Siemens, C. Domke, Ph. Ebert, and K. Urban, Phys. Rev. B 56, 12321 (1997).

${ }^{17}$ A. Sakai, H. Sunakawa, and A. Usui, Appl. Phys. Lett. 71, 2259 (1997).

${ }^{18}$ D. N. Zakharov, Z. Liniental-Weber, B. Wagner, Z. J. Reitmeier, E. A. Preble, and R. F. Davis, Phys. Rev. B 71, 235334 (2005).

${ }^{19}$ D. Hull and D. J. Bacon, Introduction to Dislocations, 4th ed (Butterworth-Heinemann, Oxford, 2001).

${ }^{20} \mathrm{~S}$. Amelinckx, in Dislocations in Solids, edited by F. R. N. Nabarro, (North-Holland, Amsterdam, 1979), Vol. 2, p. 302.

${ }^{21} \mathrm{Ph}$. Ebert, Surf. Sci. Rep. 33, 121 (1999); C. Domke, M. Heinrich, Ph. Ebert, and K. Urban, J. Vac. Sci. Technol. B 16, 2825 (1998); M. Heinrich, C. Domke, Ph. Ebert, and K. Urban, Phys. Rev. B 53, 10894 (1996)

${ }^{22}$ G. Savini, A. T. Blumenau, M. I. Heggie, and S. Öberg, Phys. Status Solidi C 4, 2945 (2007).

${ }^{23} \mathrm{Ph}$. Ebert, C. Domke, and K. Urban, Appl. Phys. Lett. 78, 480 (2001). 